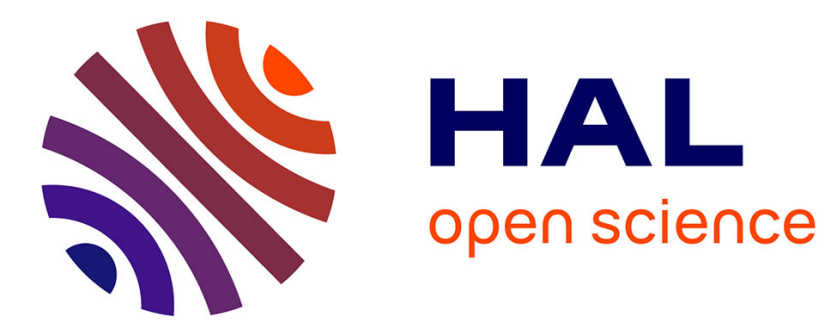

\title{
Traitement de la temporalité des discours : une Analysis Situs
}

Sylviane R. Schwer

\section{To cite this version:}

Sylviane R. Schwer. Traitement de la temporalité des discours: une Analysis Situs. 2006. halshs00085150

\section{HAL Id: halshs-00085150 \\ https://shs.hal.science/halshs-00085150}

Preprint submitted on 11 Jul 2006

HAL is a multi-disciplinary open access archive for the deposit and dissemination of scientific research documents, whether they are published or not. The documents may come from teaching and research institutions in France or abroad, or from public or private research centers.
L'archive ouverte pluridisciplinaire HAL, est destinée au dépôt et à la diffusion de documents scientifiques de niveau recherche, publiés ou non, émanant des établissements d'enseignement et de recherche français ou étrangers, des laboratoires publics ou privés. 


\title{
Traitement de la Temporalité des Discours : une Analysis Situs
}

\author{
Sylviane R. SCHWER \\ LaLICC UMR 8139 (Université Paris-Sorbonne, CNRS) et \\ LIPN UMR 7030 (Université Paris-Nord, CNRS)
}

\section{Introduction}

Une communauté importante de linguistes considère que le langage réfère in fine à des phénomènes du monde réel qui ont, en tant que tels, une extension temporelle, qu'il convient de faire coïncider par une série d'opérations cognitives, avec la représentation newtonienne du temps physique, celle de la droite géométrique. Cette démarche est connue sous le nom d'approche référentielle (Moeschler 1998, Saussure 2003). Le traitement des relations temporelles entre procès - représentations linguistiques des objets du monde réel - nécessite en premier lieu le repérage dans le texte de ces procès, ainsi que les informations qui permettent de les situer localement, sinon dans le temps, au moins relativement les uns aux autres. Il s'agit de répertorier et d'analyser les marqueurs temporels du texte. Cette tâche est purement linguistique et de grande envergure, car quasiment toutes les parties du discours contiennent des éléments utilisables comme marqueurs temporels. En second lieu, cela nécessite un système de calcul permettant la fusion de ces informations pour obtenir les situations temporelles globales. On trouve ce genre de systèmes de calcul dans les problèmes de planification, d'ordonnancement de tâches, de fusions d'information temporelles. En particulier, ces travaux font l'objet de recherches depuis plus d'une vingtaine d'années dans le domaine de l'Intelligence Artificielle sous le nom de représentations et traitements temporels (Bessière \& al. 1997). Ces travaux consistent en général à associer à tout objet une extension temporelle ponctuelle ou durative, voire une séquence de points et/ou d'intervalles, puis à traduire les informations temporelles concernant ces objets en relations binaires entre les extensions temporelles, comme un ensemble de relations atomiques possibles entre points et/ou intervalles (Schwalb \& Vila 1998). Le système de calcul se fonde essentiellement sur des fragments de logique classique, exprimables en termes d'algèbres de relations. Les relations 
atomiques en sont les éléments de base. Les calculs se font à l'aide de tables de calcul, comme celle de Allen (1983) qui a été exploitée en linguistique (Bras 1990). On ne peut faire de raisonnement temporel en linguistique, même au niveau purement qualitatif (i.e. situationnel), sans se référer à un événement ${ }^{1}$ particulier l'acte d'élocution, et son moment de réalisation. Les temps verbaux sont des marqueurs temporels importants qui nécessitent, selon Reichenbach (1947) pour leur analyse, non seulement la notion d'extension temporelle du procès $(E)$, mais aussi la notion de moment de réalisation $(\mathrm{S})$ et la notion de moment de référence $(\mathrm{R})$. Kamp \& Reyle (1993) utilisent quatre repères : le point d'énonciation (qui correspond à $S$, confondu avec le «maintenant»), un point de localisation temporelle (qui correspond à $\mathrm{E}$ ), un point de référence (présent dans le contexte qui soit est identique à $\mathrm{R}$ soit sert à enregistrer la progression du récit) et un point de perspective temporelle (indiquant le point par rapport auquel un procès est considéré). Gosselin (1996) manipule une batterie d'au moins quatre intervalles : trois correspondent à $\mathrm{S}, \mathrm{E}$ et $\mathrm{R}$ auxquels il adjoint des intervalles circonstanciels.

Le travail présenté ici se situe à l'interface de celui du linguiste et celui de la formalisation mathématique. A partir de l'étude de travaux linguistiques concernant la sémantique de la temporalité, nous avons mis en regard une certaine perception cognitivo-linguistique du temps et quelques définitions philosophiques du temps (Aristote, Leibniz) pour mettre en évidence les hypothèses suivantes :

(i) La temporalité linguistique qualitative n'a pas besoin du temps physique pour s'exprimer.

(ii) Quelque soit les valeurs aspectuelles des procès en jeu (ponctuelle, périodique ou itérative), le calcul se fait toujours sur des points ou des séquences de points, avec les deux relations fondamentales de simultanéité et de précédence. C'est une analysis situs, telle que Leibniz (Couturat 1901) la voulait : exprimer directement les relations de position, et par suite les configurations et les constructions.

(iii) Le formalisme des S-langages de Schwer (2002) est un cadre idoine pour l'expression formelle de la temporalité linguistique.

Après avoir montré que la temporalité linguistique peut s'exprimer sans recours au temps physique, nous présenterons le formalisme des S-langages qui permet de réaliser l'analysis situs. L'ensemble de toutes les relations temporelles possibles relatives à un ensemble de procès à analyser correspond un S-langage particulier qui peut être organisé sous forme d'un

${ }^{1}$ Un événement est tout ce qui se réalise dans le monde sensible, quelqu'en soit la durée. 
treillis (Autebert \& Schwer 2003). Nous montrerons alors que beaucoup de relations temporelles usuelles, en particulier celles issues de l'analyse des temps verbaux qui recouvrent souvent plusieurs configurations, correspondent à des parties convexes de ce treillis pour lesquelles les Slangages associés s'expriment simplement à l'aide d'opérateurs, sans nécessiter l'énumération de leurs éléments. Enfin, nous appliquerons notre démarche à une narration dont on calculera les chronologies compatibles avant de conclure sur les perspectives de développement de ce formalisme, en particulier en ce qui concerne la notion d'échelle ou de granularité temporelle.

\section{A propos de la temporalité linguistique}

Une autre conception du temps physique que celle défendue par Newton une donnée a priori à toute forme sensible - est celle proposée par Leibniz pour lequel « le temps n'est rien d'autre que l'ordre des événements qui se réalisent, ou bien l'ordre dans lequel les états du monde se succèdent ». C'est la voie suivie par le courant des philosophes de la nature tels Whitehead (1920), Russell (1929). Ces philosophes considèrent que l'espace et le temps sont des objets construits. Il y a un temps parce qu'il y a des choses qui arrivent, et hors des choses qui arrivent, il n'y a rien. Les événements ${ }^{2}$ dont nous avons conscience ne sont pas rigoureusement instantanés. « L'expérience immédiate ne nous fournit que des événements ordonnés par des relations de concomitance et de précédence » (Russell 1928). Les instants ne sont donc pas parmi nos données de l'expérience. Ils sont construits à partir des événements et de ces deux relations temporelles. Ces instants délimitent les durées associées aux événements comme des frontières, qui d'une part séparent un avant et un après et d'autre part assure le passage entre cet avant et cet après. Le modèle événementiel de Kamp (1979) est fondé sur cette approche.

L'image du flux des faits saillants de Gosselin (1996:51-53) réfère à une approche similaire : «le schéma cognitif du changement qui modélise le flux des événements qui se présentent est donc conçu, dès le traitement perceptif ${ }^{3}$, comme une succession de situations et de changements ... Le propre de la perception est de rendre

${ }^{2}$ «l'hypothèse fondamentale élaborée au cours de cette enquête est que les faits ultimes de la nature, dans les termes desquels toute explication physique et biologique doit être exprimée, sont des événements liés par leurs relations spatio-temporelles, et que ces relations sont dans l'ensemble réductibles à cette propriété qu'ont les événements de pouvoir contenir (ou s'étendre sur) d'autres événements qui en sont des parties » (Whitehead 1920).

${ }^{3}$ Souligné par nous. C'est sur ce traitement que nous supposons qu'est fondée la description linguistique, et non sur les propriétés physiques du temps (qui ne font pas l'unanimité parmi les physiciens). 
saillants certains des changements appartenant au flux continu des sensations. Le langage représente cette saillance perceptive selon des degrés divers. Ainsi, le fait d'être malade n'implique nullement au plan référentiel, l'absence de tout changement; simplement ils ne sont pas exprimés par le prédicat être malade. Seuls le début et la fin de la situation apparaissent comme des changements "».

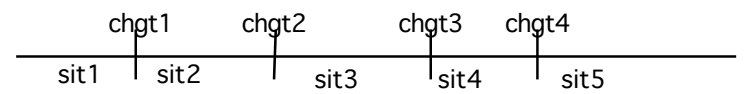

Figure 1: successions de situations et changements

Un fait saillant définit une limite entre un avant et un après; deux faits saillants délimitent une période (Figure 1). La seule séquence chgt1.chgt2.chgt3.chgt4 suffit à décrire qualitativement cette temporalité, car les situations sont entièrement définies par les changements qui constituent leur(s) bornes. Rappelons (Balaudé \& Wolff 2005) que, pour Aristote, le temps est au mouvement ce que l'espace est au corps. Sa définition du temps est « le nombre du mouvement selon l'antérieur et postérieur». Les seules propriétés du temps que l'on peut concevoir ne le sont que par dérivation de propriétés spatiales. Ce sont des propriétés qualitatives ou topologiques (ordre, continuité et limitation) et quantitatives ou métriques (additivité, mesurage). Dans cet article, seules les propriétés qualitatives nous intéressent: linéarité et continuité. La continuité du temps l'est en deux sens: (i) comme la division en puissance à l'infini, qui définit l'instant (donc en acte l'instant a une épaisseur, comme le disent les Stoïciens) (ii) comme un tout unitaire dans lequel les limites par lesquelles deux parties du temps se touchent se fondent en une seule limite. Dans sa Physique, Aristote écrit: le temps est continu par l'instant et se divise par l'instant [IV, 11, 220a5]. Mais l'instant n'est pas une partie du temps [IV, 11, 220a19]. L'instant est d'une part division du temps en puissance et d'autre part limite et unité des deux parties du temps [IV, 13, 222a18]. Un instant est à la fois le début d'une partie du temps et la fin d'une autre partie [IV, 13, 222a12]. L'intermédiaire entre deux instants est toujours un temps [VI, 1, 231b10].

Il suffit donc de donner la séquence des limites pour définir le temps, pour peu que les limites, comme les bornes des chemins, contiennent assez d'informations sur ce qui les a créées (l'ensemble des changements qui se réalisent en ce lieu temporel) et sur les parties qu'elles délimitent (les situations). Entre deux bornes différentes, on peut en ajouter autant qu'il le faut grâce à la continuité du temps. Un procès est décrit par la suite des faits saillants que le texte lui attribue. Chaque fait saillant est marqué par une occurrence de son identité. Une borne est constituée de l'ensemble des occurrences d'identités de procès correspondant aux faits saillants simultanés, c'est un instant leibnizien. Si un fait saillant $\mathrm{C}$ a été perçu avant un fait saillant $C^{\prime}$, la borne marquant $C$ précède la borne marquant $C^{\prime}$ dans la 
séquence. Dans son esprit, cette notation est très proche de la représentation diagrammatique de Reichenbach (1947) : nous allons simplement supprimer les deux signes de ponctuations, «,» pour la simultanéité et «-» pour la précédence, et utiliser une représentation faisant appel à la notion de borne. Examinons l'exemple (1) de (Reichenbach 1947).

(1) «I had mailed the letter (P1) when John came (P2) and told me the news $(\mathrm{P} 3)$ » (j'avais déjà envoyé la lettre (P1) quand Jean vint (P2) et me donna les informations (P3)).

Selon le modèle de Reichenbach, le procès $\mathrm{P} 1$ correspond au diagramme E1$\mathrm{R} 1-\mathrm{S}^{4}$, le second à $\mathrm{R} 2, \mathrm{E} 2-\mathrm{S}$ et le troisième à $\mathrm{R} 3, \mathrm{E} 3-\mathrm{S}$ puis il traduit la règle grammaticale «c'est le même point de référence pour les trois événements » par: R1,R2,R3, ce qui donne le diagramme global E1-R1,R2,R3,E2,E3-S. Prenons comme identité le nom des objets, E1, E2 et E3 pour les procès, R pour l'unique point de référence (donc identité unique), et $\mathrm{S}$ pour l'acte d'énonciation. Notons $\Xi$ l'opérateur de fusion des informations. La description s'exprime par l'expression E1RS $\Xi\{R, E 2\} S \Xi\{R, E 3\} S$; son résultat vaut $\mathrm{E} 1\{\mathrm{R}, \mathrm{E} 2, \mathrm{E} 3\} \mathrm{S}$, qui détermine trois bornes ou moments: le premier correspond à l'occurrence de E1, le second moment aux occurrences simultanées de E2 et E3 et le troisième moment à l'occurrence du moment de la parole. Rien n'est dit concernant le temps écoulé entre ces moments. Nous rendons simplement compte ici du fait que le temps est bien construit à partir des événements (linguistiques), suivant en cela la conception du temps selon Leibniz.

\section{Le modèle formel des S-langages.}

Il s'agit de généraliser la description précédente du modèle de Reichenbach. A chaque objet $\mathrm{P}$ pour lequel on pose une extension temporelle, notamment les procès, nous associons une identité $p$. L'ensemble des identités des objets en jeu est pris comme alphabet sur lequel on va les décrire ainsi que leurs relations temporelles. La description d'un procès consiste à représenter l'extension temporelle qu'on lui accorde, en termes de bornes. Un procès considéré comme ponctuel est décrit par le mot l'une seule lettre $a$; considéré comme duratif c'est le mot de deux lettres aa qui le décrira.

\subsection{Définition d'un S-alphabet, d'un S-mot, d'un S-langage .}

Nous utilisons les notions de base de la théorie des langages, telles que présentées par Autebert (1987). Soit X un ensemble fini appelé alphabet dont les éléments sont appelés lettres. Un mot est une séquence finie de lettres, un

\footnotetext{
${ }^{4}$ E représente l'instant de l'événement, $\mathrm{R}$ celui du point de référence (d'où l'on saisit l'événement) et S l'instant de la parole.
} 
sous-mot $h$ d'un mot $f$ est une séquence extraite de la séquence $f$ : le mot $b b c$ est un sous-mot du mot $a b c b a c$, écrit sur l'alphabet $\mathrm{X}=\{\mathrm{a}, \mathrm{b}, \mathrm{c}\}$. L'ensemble des parties non vides de X, est le $S$-alphabet noté $\hat{X}$. On identifie toute $S$ lettre singleton $\{\mathrm{x}\}$ avec $x,(X \subseteq \hat{X})$. Un $S$-mot (sur X) est une séquence finie de S-lettres, un S-langage un ensemble de S-mots. L'ensemble de tous les Smots sur $X$ est noté $\hat{X}^{*}$. soit l'alphabet $X=\left\{\mathrm{x}_{1}, \ldots, \mathrm{x}_{\mathrm{n}}\right\}$, on peut diviser $\hat{X}^{*}$ en parties $\Delta^{X}\left(d_{1}, \ldots, d_{n}\right)$, ensemble de tous les S-mots comportant pour tout indice $i$ exactement $d i$ occurrences de la lettre $x_{i}$. Ainsi pour $\mathrm{X}=\{\mathrm{p}, \mathrm{q}\}$, $\Delta^{\mathrm{X}}(1,1)=\{\mathrm{pq},\{\mathrm{p}, \mathrm{q}\}, \mathrm{qp}\}$ code les positions relatives possibles de deux points ;

$\Delta^{\mathrm{x}}(1,2)=\{\mathrm{pqq},\{\mathrm{p}, \mathrm{q}\} \mathrm{q}, \mathrm{qpq}, \mathrm{q}\{\mathrm{p}, \mathrm{q}\}, \mathrm{qqp}\}$ code les positions relatives possibles entre un point $A$ et un intervalle (ou une séquence de deux points) B.

$\Delta^{\mathrm{x}}(2,2)=\{\mathrm{ppqq}, \mathrm{p}\{\mathrm{p}, \mathrm{q}\} \mathrm{q}, \mathrm{pqpq},\{\mathrm{p}, \mathrm{q}\} \mathrm{pq}, \mathrm{pq}\{\mathrm{p}, \mathrm{q}\},\{\mathrm{p}, \mathrm{q}\}(\mathrm{p}, \mathrm{q}\}, \mathrm{qppq}, \mathrm{pqqp}, \mathrm{qp}\{\mathrm{p}, \mathrm{q}\}$, $\{\mathrm{p}, \mathrm{q}\} \mathrm{qp}, \mathrm{qpqp}, \mathrm{q}\{\mathrm{p}, \mathrm{q}\} \mathrm{p}$, qqpp $\}$ permet de coder l'ensemble des positions relatives possibles (i) entre deux intervalles, (ii) entre un intervalle et une séquence de deux points (ou réciproquement), (iii) entre deux séquences de deux points. C'est en particulier le S-langage associé aux relations atomiques de Allen (1983).

Pour $X=\{e, r, s\}, \Delta^{X}(1,1,1)=\{$ ers, $\{e, r\} s, e\{r, s\}$, res, esr, $\{e, r, s\}, r\{e, s\},\{e, s\} r$, rse, ser, $(\mathrm{r}, \mathrm{s}\} \mathrm{e}, \mathrm{s}\{\mathrm{e}, \mathrm{r}\}$, sre $\}$ code l'ensemble des positions relatives entre trois points, qui correspond au S-langage de Reichenbach pour décrire les temps verbaux.

\subsection{Les S-opérateurs}

En dehors des opérateurs classiques des langages formels comme les opérations ensemblistes (concaténation, union, intersection, différence) et les morphismes directs et inverses, nous utilisons trois opérateurs nouveaux : la S-substitution, la S-projection et la S-jointure.

Les opérateurs classiques des langages formels traitent les S-lettres comme des objets atomiques, ils n'opèrent pas à l'intérieur. Les S-opérations opèrent à l'intérieur des S-lettres.

La S-substitution permet de renommer à l'intérieur d'un S-mot, toutes les occurrences d'une même lettre et, par élimination des répétitions dans une Slettre, de produire un nouveau S-mot. Dans le S-mot $\{a, b\} c$, le renommage de la lettre b par la lettre a donne le S-mot $\{a\} c$. Ce même renommage transforme $\Delta^{\{a, b, c\}}(1,1,1)$ en $\Delta^{\{a, c\}}(2,1)$.

La S-projection d'un alphabet X sur un sous-alphabet Y est une opération qui, prenant un S-mot $f$ écrit sur l'alphabet $\mathrm{X}$, produit sur l'alphabet $\mathrm{Y}$, après élimination des S-lettres devenues vides, le S-mot $f \mid Y$ obtenu par effacement de toutes les occurrences de lettres qui ne font pas partie de l'alphabet $\mathrm{Y}$. La projection de l'alphabet $\mathrm{X}=\{\mathrm{a}, \mathrm{b}, \mathrm{c}\}$ sur l'alphabet $\mathrm{Y}=\{\mathrm{a}, \mathrm{b}\} \mathrm{du}$ S-mot 
$\mathrm{f}=\{\mathrm{a}, \mathrm{c}\}\{\mathrm{a}, \mathrm{b}\}\{\mathrm{c}, \mathrm{d}\}\{\mathrm{a}, \mathrm{b}, \mathrm{c}\}$ est le S-mot $\mathrm{f} \mid \mathrm{Y}=\mathrm{a}\{\mathrm{a}, \mathrm{b}\}\{\mathrm{a}, \mathrm{b}\}$; la S-projection de $\Delta^{(\mathrm{a}, \mathrm{b}, \mathrm{c}\}}(1,1,1)$ est $\Delta^{(\mathrm{a}, \mathrm{b}\}}(1,1)$.

Le $S$-mélange, noté $\otimes$, de deux S-mots dont les alphabets sont disjoints est l'ensemble de toutes les combinaisons que l'on peut faire avec ces deux Smots. Par exemple $\mathrm{a} \otimes \mathrm{b}=\{\mathrm{ab},\{\mathrm{a}, \mathrm{b}\}, \mathrm{ba}\}=\Delta^{\{\mathrm{a}, \mathrm{b}\}}(1,1), \mathrm{ac} \otimes \mathrm{b}=\{\mathrm{acb}, \mathrm{a}\{\mathrm{c}, \mathrm{b}\}, \mathrm{abc},\{\mathrm{a}, \mathrm{b}\} \mathrm{c}$, $\mathrm{bac}\}, \mathrm{a} \otimes \mathrm{bb}=\Delta^{\{\mathrm{a}, \mathrm{b}\}}(1,2), \mathrm{aa} \otimes \mathrm{bb}=\Delta^{\{\mathrm{a}, \mathrm{b}\}}(2,2)$.

L'opération de $S$-jointure, notée $\Xi$ est l'opération essentielle pour le calcul. C'est une opération dont le résultat est un S-langage, i.e. un ensemble de Smots. Soient $f$ et $g$ les deux S-mots à joindre. Prenons un alphabet $\mathrm{X}$ possédant au moins toutes les lettres des mots $\mathrm{f}$ et $\mathrm{g}$. Notons $\mathrm{X} \mid \mathrm{f}[\mathrm{resp} . \mathrm{X} \mid \mathrm{g}]$ le sous-alphabet de $\mathrm{X}$ constitué uniquement des lettres intervenant dans le mot $f[$ resp. $G]$. Considérons l'ensemble des lettres communes à X $\mid \mathrm{f}$ et $\mathrm{X} \mid \mathrm{g}$.

Si cet ensemble est vide, alors $\mathrm{f} \Xi \mathrm{g}=\mathrm{f} \otimes \mathrm{g}$.

Si cet ensemble est non vide, ignorons alors dans chacun des mots, toutes les autres lettres. Deux cas peuvent se produire : (i) les deux sous-mots obtenus coïncident (ii) les deux sous-mots ne coïncident pas.

Pour le mot $f=\{\mathrm{a}, \mathrm{c}\}\{\mathrm{a}, \mathrm{b}\}\{\mathrm{c}, \mathrm{d}\}\{\mathrm{a}, \mathrm{b}, \mathrm{c}\}, \mathrm{X} \mid \mathrm{f}=\{\mathrm{a}, \mathrm{b}, \mathrm{c}, \mathrm{d}\}$, pour le mot $\mathrm{g}=\mathrm{x}\{\mathrm{a}, \mathrm{x}, \mathrm{y}\} \mathrm{x}\{\mathrm{a}, \mathrm{b}\} \mathrm{y}$ $\{a, b, y\} x, X \mid g=\{a, b, x, y\}$, pour le mot $h=\{a, x, y\} x\{a, b\} y X \mid h=\{a, b, x, y\}$. L'alphabet des lettres communes est $\mathrm{Z}=\{\mathrm{a}, \mathrm{b}\}$. La projection de $f$ sur $Z$ est $\left.\mathrm{f}\right|_{\mathrm{Z}}=\mathrm{a}\{\mathrm{a}, \mathrm{b}\}\{\mathrm{a}, \mathrm{b}\}$, la projection de $g$ sur $Z$ est $\mathrm{g}_{\mathrm{Z}}=\mathrm{a}\{\mathrm{a}, \mathrm{b}\}\{\mathrm{a}, \mathrm{b}\}$, la projection de $h$ sur $\mathrm{Z}$ est $\left.\mathrm{h}\right|_{\mathrm{Z}}=\mathrm{a}\{\mathrm{a}, \mathrm{b}\}$. $\left.\mathrm{f}\right|_{Z}=\left.\mathrm{g}\right|_{Z}$ qui correspond au cas (i), $\left.\mathrm{f}\right|_{Z} \neq\left.\mathrm{h}\right|_{Z}$, ce qui correspond au cas (ii).

La S-jointure de deux mots satisfaisant le cas (ii) est le langage vide.

La S-jointure de deux S-mots $f$ et $g$ satisfaisant le cas (i) est le S-langage constitué de tous les S-mots écrits sur l'union des alphabets $X \mid \mathrm{f}$ et $\mathrm{X} \mid \mathrm{g}$ qui satisfont la propriété suivante : leur S-projection sur $\mathrm{X} \mid \mathrm{f}$ égale $\mathrm{f}$ et leur $\mathrm{S}$ projection sur $\mathrm{g}$ égale $\mathrm{g}$.

Formellement, en notant T l'union des alphabets $\mathrm{X} \mid \mathrm{f}$ et $\mathrm{X} \mid \mathrm{g}$, la jointure des deux mots $f$ et $g$ vaut $f \Xi g=\left\{h \in \hat{T}^{*},\left(h_{|X| f}=f\right)\right.$ et $\left.\left(h_{|X| g}=g\right)\right\}$.

Quand les alphabets X|f et X|g sont disjoints, le cas (i) s'applique. Le Smélange est donc un cas particulier de la $\mathrm{S}$-jointure qui correspond à l'absence de contrainte temporelle.

Ces quatre opérations s'étendent à des opérations sur les S-langages, la Sprojection et la S-substitution s'appliquent à chaque S-mot du S-langage, la S-jointure [resp. S-mélange] de deux S-langages $\mathrm{M}$ et $\mathrm{N}$ est le S-langage [resp. S-mélange] union des S-jointures [resp. S-mélanges] d'un S-mot de M et d'un S-mot de N. La S-jointure [resp. S-mélange] est une opération associative, commutative sur les S-langages d'alphabet $\mathrm{X}$, avec pour élément neutre.

\subsection{Algorithme de calcul.}


Décrivons l'algorithme de construction de la jointure sur les deux mots $f$ et $g$ considérés précédemment, dont nous avons souligné les lettres communes : $\mathrm{f}=\{\underline{\mathrm{a}}, \mathrm{c}\}\{\underline{\mathrm{a}}, \underline{\mathrm{b}}\}\{\mathrm{c}, \mathrm{d}\}\{\underline{\mathrm{a}}, \underline{\mathrm{b}}, \mathrm{c}\}$ et $\mathrm{g}=\mathrm{x}\{\underline{\mathrm{a}}, \mathrm{x}, \mathrm{y}\} \mathrm{x}\{\underline{\mathrm{a}}, \underline{\mathrm{b}}\} \mathrm{y}\{\underline{\mathrm{a}}, \underline{\mathrm{b}}, \mathrm{y}\} \mathrm{x}$. L'alphabet commun est $\mathrm{Z}=\{\underline{\mathrm{a}}, \underline{\mathrm{b}}\}$ et 1 'alphabet conjoint est $\mathrm{T}=\{\underline{\mathrm{a}}, \underline{\mathrm{b}}, \mathrm{c}, \mathrm{d}, \mathrm{x}, \mathrm{y}\}$. Le S-mot commun $\underline{\mathrm{a}}\{\underline{\mathrm{a}}, \underline{\mathrm{b}}\}\{\underline{\mathrm{a}}, \underline{\mathrm{b}}\}=\mathrm{f}|\mathrm{Z}=\mathrm{g}| \mathrm{Z}$. Leur S-jointure est obtenue de la façon suivante :

- pour chaque occurrence de lettre commune, fusionner les deux S-lettres les contenant et les disposer dans l'ordre de lecture. Il y a trois couples de Slettres concernées : $(\{\underline{\mathrm{a}}, \mathrm{c}\},\{\underline{\mathrm{a}}, \mathrm{x}, \mathrm{y}\}),(\{\underline{\mathrm{a}}, \underline{\mathrm{b}}\},\{\underline{\mathrm{a}}, \underline{\mathrm{b}}, \mathrm{y}\})$ et $(\{\underline{\mathrm{a}}, \underline{\mathrm{b}}, \mathrm{c}\},\{\underline{\mathrm{a}}, \underline{\mathrm{b}}, \mathrm{y}\})$. La fusion par paire (ie l'union) de ces S-lettres donne la séquence $\{\underline{\mathrm{a}}, \mathrm{c}, \mathrm{x}, \mathrm{y}\}\{\underline{\mathrm{a}}, \underline{\mathrm{b}}, \mathrm{y}\}(\underline{\mathrm{a}}, \underline{\mathrm{b}}, \mathrm{c}, \mathrm{y}\}$.

- les occurrences des lettres non communes doivent être placées en respectant l'ordre interne au S-mot qui les contient. Devant la première S-lettre, derrière la dernière $\mathrm{S}$-lettre et entre (devant la première, derrière la dernière) deux $\mathrm{S}$ lettres contenant des lettres communes, plaçons les S-lettres comprises entre les deux S-lettres (devant la première, derrière la dernière) correspondantes de $\mathrm{f}$ et $\mathrm{g}$, cela de toutes les façons possibles car il n'y a aucun indice pour les ordonner. On utilise alors le S-mélange pour exprimer la solution : $\{\underline{\mathrm{a}}, \mathrm{c}\}\{\underline{\mathrm{a}}, \underline{\mathrm{b}}\}\{\mathrm{c}, \mathrm{d}\}\{\underline{\mathrm{a}}, \underline{\mathrm{b}}, \mathrm{c}\} \otimes \mathrm{x}\{\underline{\mathrm{a}}, \mathrm{x}, \mathrm{y}\} \mathrm{x}\{\underline{\mathrm{a}}, \underline{\mathrm{b}}\} \mathrm{y}\{\underline{\mathrm{a}}, \underline{\mathrm{b}}, \mathrm{y}\} \mathrm{x}=\mathrm{x}\{\mathrm{a}, \mathrm{c}, \mathrm{x}, \mathrm{y}\} \mathrm{x}\{\mathrm{a}, \mathrm{b}\} \cdot[\{\mathrm{c}, \mathrm{d}\} \otimes \mathrm{y}]\{\mathrm{a}, \mathrm{b}, \mathrm{c}$, $\mathrm{y}\} \mathrm{x}$. Le S-mélange $[\{\mathrm{c}, \mathrm{d}\} \otimes \mathrm{y}]$ égale l'ensemble des trois S-mots $\{\mathrm{c}, \mathrm{d}\} \mathrm{y},\{\mathrm{c}, \mathrm{d}, \mathrm{y}\}$ et $\mathrm{y}\{\mathrm{c}, \mathrm{d}\}$.

Le traitement informatique du raisonnement temporel est fondé sur la proposition suivante : il existe une expression explicite (et régulière) de $\mathrm{f} \Xi \mathrm{g}$. Pour faciliter le calcul sur les S-langages et en réduire la complexité, la notion de S-langage élémentaire a été introduite (Battistelli \& al 2004) et définie récursivement comme suit : un S-mot est un S-langage élémentaire, un S-langage élémentaire s'exprime uniquement à l'aide de S-mélanges et de produits de S-langages élémentaires. Soit sur l'alphabet $\{a, b, c\}$, le S-langage $\{(\mathrm{a}, \mathrm{b}) \mathrm{abcc} ;(\mathrm{a}, \mathrm{b})(\mathrm{a}, \mathrm{b}) \mathrm{cc} ;(\mathrm{a}, \mathrm{b}) \mathrm{bacc} ;(\mathrm{a}, \mathrm{b}) \mathrm{b}(\mathrm{a}, \mathrm{c}) \mathrm{c} ;(\mathrm{a}, \mathrm{b}) \mathrm{bcac}\}$ est un S-langage élémentaire car il peut s'exprimer sous la forme: $(a, b)[a \otimes b] c$. La S-jointure calculée précédemment est également un S-langage élémentaire. De même, le S-langage $\{a b a b,\{a, b\} a b, a b\{a, b\}, a b b a,\{a, b\}\{a, b\}, b a a b, b a\{a, b\},\{a, b\} b a, b a b a\}$ qui représente $\mathrm{A} 0 \mathrm{~B}$, la relation de chevauchement des événements de Kamp, est aussi un S-langage élémentaire car il s'exprime par $[a \otimes b][a \otimes b]$.

Le S-mélange est le cas extrême de la S-jointure. Il permet, une fois la représentation des objets temporels déterminés, d'exprimer ou de calculer l'ensemble de toutes les situations temporelles possibles existant entre elles. Nous avons déjà vu que $\Delta^{(\mathrm{R}, \mathrm{S}, \mathrm{T}\}}(1,1,1)$ correspond au modèle de Reichenbach et $\Delta^{(\mathrm{z}, \mathrm{b})}(2,2)$ à celui des situations temporelles possibles entre deux périodes.

En confondant un S-langage ne contenant qu'un seul S-mot avec ce S-mot, on peut remarquer que $\mathrm{x}_{1}{ }^{\mathrm{d} 1} \otimes \ldots \otimes \mathrm{x}_{\mathrm{n}}{ }^{\mathrm{dn}}=\Delta^{\{\mathrm{x} 1, \ldots, \mathrm{xn}\}}\left(\mathrm{d}_{1}, \ldots, \mathrm{d}_{\mathrm{n}}\right)$. Cette famille de S- 
langages, que nous nommons S-langage univers, est en correspondance naturelle avec l'ensemble des chemins de Delannoy $\Delta\left(\mathrm{p}_{1}, \ldots \mathrm{p}_{\mathrm{n}}\right)$ (Schwer 2002), qui définit le type du S-langage et permet de calculer le nombre de Smots dans une S-jointure à partir de la connaissance de celui des chemins de Delannoy. Pour coder des intervalles, on utilise des langages du type $\Delta(2, \ldots, 2)$. Pour deux intervalles, il y a 13 S-mots, pour trois intervalles, il y en a 409 ! Pour coder des points, on utilise des langages du type $\Delta(1, \ldots, 1)$. Pour trois points, il y a $13 \mathrm{~S}$-mots, pour quatre points, 75 S-mots. Du point de vue du calcul, le choix de la modélisation des procès n'est pas anodin, car associer des intervalles plutôt que des points à des procès, quand il s'agit de simplement les situer les uns par rapport aux autres, induit un espace de calcul bien plus important. En effet, puisqu'il s'agit de situer les procès les uns par rapport aux autres, le fait d'utiliser une représentation ponctuelle pour représenter un procès au passé simple, n'indique pas que le procès est ponctuel, mais simplement qu'il est considéré comme atomique, au sens où il ne contient pas d'autres procès.

D'autre part, Autebert \& Schwer (2003) ont montré que ces ensembles sont organisés en treillis ${ }^{5}$. Cette notion est importante pour l'expression des relations temporelles en linguistique car c'est très souvent en ces termes qu'elles s'expriment, les informations temporelles étant souvent sousspécifiées. Ces parties convexes correspondent à des S-langages élémentaires. Tout S-langage peut s'exprimer comme une union de Slangages élémentaires. Le problème est de trouver une expression minimale, i.e. de minimiser le nombre de S-langages élémentaires constituant le Slangage. Il s'agit d'étudier les parties convexes du S-langage dans l'ensemble des situations possibles organisé en treillis. C'est également sous cette forme que le linguiste attend la restitution du résultat de son calcul et non par une définition en extension de l'ensemble de toutes les situations temporelles possibles, libre à lui ensuite de choisir soit cette expression globale, soit une solution type (Gosselin 1996), ou solution par défaut (Battistelli 2000, Saussure 2003) parmi les solutions possibles. L'organisation en treillis de $\Delta^{\left(\mathrm{R}, \mathrm{S}, \mathrm{T}_{3}\right.}(1,1,1)$, qui correspond au modèle de Reichenbach et de $\Delta^{\left(\mathrm{z}, \mathrm{b}_{3}\right.}(2,2)$ qui correspond à celui des situations temporelles possibles entre deux périodes sont représentées dans la figure suivante.

- Kamp \& Reyle (1993) utilisent un S-langage domaine de type $\Delta(1,1,1,1)$.

- Dermidache \& Uribe-Etxebarria (2002) utilisent des périodes pour décrire les extensions temporelles de l'événement(e) et de l'assertion(a) et un point pour le moment d'énonciation $(u)$, ce qui utilise le S-langage univers $\Delta^{\{e, a, u\}}(2,2,1)$. Leur compréhension du passé progressif anglais

(2) « Naïma was reading Ramza»

${ }^{5}$ Un treillis est un ensemble ordonné dans lequel deux éléments quelconques ont un plus grand élément inférieur commun et un plus petit élément supérieur commun. 
se traduit par le $\mathrm{S}$-langage eaa[ $[\mathrm{e} \otimes \mathrm{u}]$ qui décrit l'absence d'information situationnelle entre la fin de l'événement et le moment d'énonciation.

- Vet (2004) utilise les trois repères de Reichenbach, en introduisant un repère supplémentaire, le point de perspective (qu'il substitue parfois à R) et en adjoignant au repère de l'événement $E$ parfois sa phase préparatoire (E') et parfois sa phase résultative (E"). Tous les temps verbaux n'appartiennent donc pas au même Slangage univers dont le type est soit $\Delta(1,1,1)$ soit $\Delta(1,1,1,1)$ soit $\Delta(1,1,1,1,1)$.

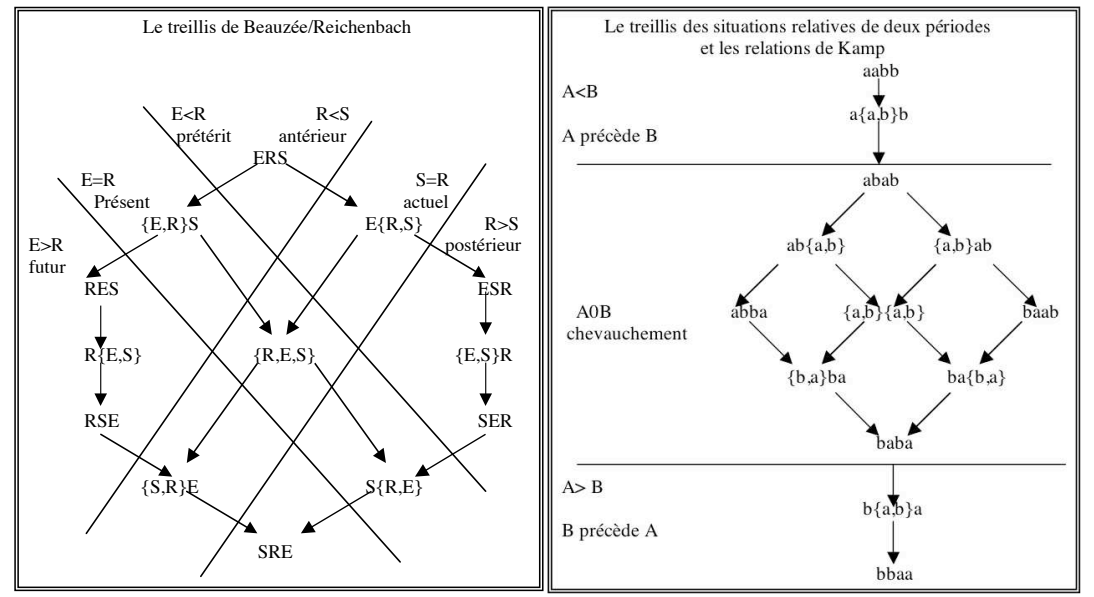

Figure 2 : Les treillis de deux S-langages domaines

\section{Applications}

Nous avons travaillé principalement sur le S-mélange, qui correspond à l'absence totale d'information temporelle. Une S-jointure vide est l'autre cas extrême, qui traduit une contradiction temporelle. Une S-jointure réduite à un seul mot traduit une information temporelle complète. Les autres S-jointures traduisent une sous-détermination des informations.

\subsection{Recueil de témoignages}

L'une des applications importante du raisonnement temporel en Intelligence Artificielle consiste à déterminer l'ensemble des situations temporelles possibles cohérentes avec un ensemble d'informations issues de différentes sources. Un cas typique est celui du recueil de témoignages au cours d'une enquête policière. Si l'adage " testus unus testus nullus » relève d'un principe de précaution, le traitement des témoignages multiples peut conduire à des 
contradictions, souvent révélées par des incohérences temporelles ${ }^{6}$. Deux attitudes sont alors possibles : soit prendre l'union de toutes les informations, soit prendre l'intersection commune des informations. La seconde attitude correspond à l'opération de S-jointure. Nous allons étudier quatre énoncés différents pour nous permettre de jouer avec la représentation en S-mots, puis nous travaillerons d'une part sur les deux premiers énoncés qui sont clairement contradictoires et sur les deux derniers qui sont différents mais pas contradictoires. Nous cherchons ici à modéliser un raisonnement spatiotemporel, au travers d'une séquence d'énoncés rendant compte des compatibilités de positionnements d'entités dans l'espace et dans le temps en tenant compte de la sémantique du temps et de la sémantique verbale.

Considérons les quatre énoncés suivant :

(2) «Eve est venue avant Joe»

(3) «Eve est venue après Joe »,

(4) «Eve a rencontré Joe»

(5) « Joe est arrivé avant Eve»

Associons à la localisation spatiale statique de Eve «Etrelà(Eve)» le mot $e e$ et le mot $j j$ à la localisation spatiale statique de Jean «Etrelà(Joe)» et traduisons en S-langage les quatre énoncés en supposant que « est venue avant/après » exclut le fait d'être-là ensemble. (2) donne une information complète, son S-langage L2 se réduit au seul S-mot eejj ${ }^{7}$. De même l'énoncé (3) correspond au S-langage L3=jjee. L'énoncé (4) est sous-spécifié, il correspond à l'opérateur de chevauchement de Kamp dont le S-langage est $\mathrm{L} 4=[\mathrm{j} \otimes \mathrm{e}][\mathrm{j} \otimes \mathrm{e}]$, qui signifie qu'aucune information ne permet de situer relativement ni les bornes initiales ni les bornes finales, mais que les bornes initiales précèdent les bornes finales (ce qui laisse une plage de rencontre). L'énoncé (5) est également sous-spécifié, puisque qu'il permet de situer la borne initiale du procès $J$ devant la borne initiale du procès $E$ mais rien n'est dit concernant les bornes finales, ce qui correspond au S-langage $\mathrm{L} 5=\mathrm{j}[\mathrm{j} \otimes e e]$. Le S-langage associé à l'ensemble des énoncés est contenu dans la S-jointure des S-langages de chacun des énoncés. Il y a inclusion et non égalité, car des indices discursifs ou pragmatiques peuvent enrichir la connaissance de la temporalité globale, ce qui peut éliminer certains S-mots.

\footnotetext{
${ }^{6}$ Qui n'ont rien à voir avec les lois de la théorie de la relativité, qui peut effectivement conduire deux observateurs à voir l'occurrence de deux événements A et B dans des ordres différents, car la physique classique s'applique de façon performante à l'échelle des événements humains.

${ }^{7}$ Dans ce cas, on confond le S-mot avec le S-langage réduit à ce S-mot. On écrit $\mathrm{L} 2=\mathrm{eejj}$
} 
Considérons les deux énoncés (2) et (3) ensemble, le S-langage associé à cet ensemble est $\mathrm{L} 2 \Xi \mathrm{L} 3=\mathrm{eejj} \Xi \mid \mathrm{jjee}=\varnothing$, ce qui traduit bien une contradiction temporelle de l'ensemble. Quant aux deux énoncés (4) et (5), leur ensemble correspond au S-langage L4 $\Xi \mathrm{L} 5=[\mathrm{j} \otimes \mathrm{e}][\mathrm{j} \otimes \mathrm{e}] \Xi \mid \mathrm{j}[\mathrm{j} \otimes \mathrm{ee}]=\mathrm{je}[\mathrm{j} \otimes \mathrm{e}]$. En effet, L5 exige que la borne initiale du procès $J$ soit temporellement la première borne et L4 exige que les deux bornes initiales précèdent les deux bornes finales, ce qui positionne la borne initiale du procès $\mathrm{E}$ en deuxième position. Mais aucune information ne permet de positionner les bornes finales. Ainsi, les deux énoncés (4) et (5) peuvent être validés simultanément, ils correspondent aux trois situations possibles données Figure 3.

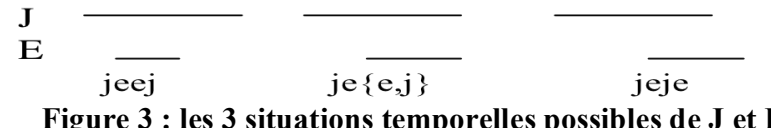

\subsection{Raisonnement temporel à partir d'un texte étiqueté}

Nous avons implanté la méthode des S-langages dans un prototype jouet (Battistelli \& al. 2004), afin d'en valider l'approche. Le modèle linguistique donné est celui de Desclés (1989) et Desclés \& Guentchéva (2006) et les règles temporelles de l'analyse linguistique adoptées celles de Battistelli (2000). Dans ce modèle, les procès sont classés en états, événements et processus, et associés à des intervalles de nombres réels, respectivement ouverts (états), fermés (événements) ou fermés à gauche et ouverts à droite (processus). Le typage des bornes empêche certaines situations temporelles de se produire. Cela impose ${ }^{8}$ la règle

(RG1) La fin d'un événement ne peut coïncider avec le début d'un événement ou d'un processus ou avec la fin d'un processus ou d'un état

Une autre règle nous a été également imposée :

(RG2) Aucun procès n'entrelace un autre procès.

L'un des textes étiquetés examinés est le suivant :

(5)Etant arrêté momentanément sur la file droite du Bd des Italiens (P1 : état), j'avais mis mon clignotant (P2 : état), j'étais à l'arrêt (P3 : état). Le véhicule $\mathrm{B}$ arrivant sur ma gauche ( $\mathrm{P} 4$ : processus) m'a serré de trop près (P5: évènement) et m'a abîmé tout le côté avant gauche (P6 : évènement).

\footnotetext{
${ }^{8}$ Le but du jeu était de modéliser les règles du modèle et les règles linguistiques sous forme de S-langages telles qu'elles étaient données par les linguistes, non d'en critiquer la pertinence. Remarquons que RG1 empêche de modéliser des expressions comme "immédiatement après » ou " aussitôt ». Au cours d'une discussion ultérieure concernant RG2, Zlatka Guentcheva a admis la possibilité de chevauchement, dans le cas où les acteurs des procès sont différents.
} 
Les contraintes temporelles linguistiques déduites de l'étude du texte sont les trois règles suivantes :

(R1) P1, P2, P3 sont contemporains et contiennent les autres procès

(R2) $\mathrm{P} 5$ commence après $\mathrm{P} 4$

(R3) P6 commence_en_même_temps_ou_après P5

Nous avons opté pour la méthode suivante : les contraintes temporelles déduites de l'étude du texte sont calculées en premier, celles du modèle utilisé sont utilisées comme filtrage sur la solution obtenue. L'avantage de ce choix dans l'usage du modèle de Desclès est que la règle d'entrelacement (RG2) - outre le fait qu'elle n'est pas admise universellement - casse systématiquement les S-langages élémentaires, qui permettent de décrire un grand nombre de contraintes temporelles exprimées par les textes, ce qui reste notre motivation première.

Description du langage : l'alphabet utilisé est $\mathrm{X}=\{\mathrm{p} 1, \mathrm{p} 2, \mathrm{p} 3, \mathrm{p} 4, \mathrm{p} 5, \mathrm{p} 6\}$. Les occurrences de ces lettres portent la nature des bornes correspondantes selon la convention suivante: les bornes fermées sont soulignées. Le Slangage univers est $\Delta^{\mathrm{x}}(2,2,2,2,2,2)=\mathrm{p} 1 \mathrm{p} 1 \otimes \mathrm{p} 2 \mathrm{p} 2 \otimes \mathrm{p} 3 \mathrm{p} 3 \otimes \mathrm{p} 4 \mathrm{p} 4 \otimes \mathrm{p} 5 \mathrm{p} 5 \otimes \mathrm{p} 6 \mathrm{p} 6$ qui contient 308682 S-mots.

Expression des règles: la règle $(\mathrm{R} 1)$ se traduit par le S-langage $\mathrm{L} 1=[\mathrm{p} 1 \otimes \mathrm{p} 2 \otimes \mathrm{p} 3][\mathrm{p} 4 \mathrm{p} 4 \otimes \mathrm{p} 5 \mathrm{p} 5 \otimes \mathrm{p} 6 \mathrm{p} 6][\mathrm{p} 1 \otimes \mathrm{p} 2 \otimes \mathrm{p} 3]$ qui contient $13 * 409 * 13$ soit $69121 \mathrm{~S}$-mots.

La règle $(\mathrm{R} 2)$ se traduit par le S-langage $\mathrm{L} 2=\mathrm{p} 4[\mathrm{p} 4 \otimes \mathrm{p} 5 \mathrm{p} 5]$, qui ne fait apparaître que les lettres $\mathrm{p} 4$ et $\mathrm{p} 5$, c'est-à-dire traduit une contrainte partielle. De même la règle $(\mathrm{R} 3)$ est associé au S-langage $\mathrm{L} 3=\{\underline{\mathrm{p} 5, \mathrm{p} 6}\}[\mathrm{p} 5 \otimes \mathrm{p} 6] \mathrm{U}$ p6 $[\mathrm{p} 5 \mathrm{p} 5 \otimes \mathrm{p} 6]$ qui ne fait apparaître que les lettres p5 et p6. Ce S-langage n'est pas élémentaire, il comporte deux S-langages élémentaires, leur union est la traduction du « soit » qui ici n'est pas réductible à une expression non disjonctive.

Le S-langage correspondant à la conjonction des trois règles est donné par l'expression L1 $\Xi$ L2 $\Xi L 3$ qui, calcul fait, donne le S-langage :

$\mathrm{L}=[\mathrm{p} 1 \otimes \mathrm{p} 2 \otimes \mathrm{p} 3] \mathrm{p} 4[\mathrm{p} 4 \otimes\{\mathrm{p} 5, \mathrm{p} 6\}[\mathrm{p} 5 \otimes \mathrm{p} 6]][\mathrm{p} 1 \otimes \mathrm{p} 2 \otimes \mathrm{p} 3] \mathrm{U}[\mathrm{p} 1 \otimes \mathrm{p} 2 \otimes \mathrm{p} 3] \mathrm{p} 4[\mathrm{p} 4$ $\otimes \mathrm{p} 5[\mathrm{p} 5 \otimes \mathrm{p} 6 \mathrm{p} 6]][\mathrm{p} 1 \otimes \mathrm{p} 2 \otimes \mathrm{p} 3]$ qui contient $13 *[19+41]^{*} 13=10140$ S-mots. Le fragment concernant le processus P4 et les deux événements P5 et P6 correspond à 60 positions possibles entre eux.

(RG1) correspond au schéma d'exclusion suivant: $\underline{x}\{\underline{x}, \underline{y}\} \underline{y}$ ni $\underline{x}\{\underline{x}, \underline{y}\}$ y ni $[\mathrm{x} \otimes \mathrm{y}]\{\mathrm{x}, \mathrm{y}\}$ (les premières occurrences de $\mathrm{x}$ et $\mathrm{y}$ étant soulignées ou non).

(RG2) correspond au schéma d'exclusion suivant: xyxy (lettres soulignées ou non).

Filtrer par ces Règles Générales consiste à exclure les S-mots possédant un de ces motifs. Nous donnons une expression du S-langage résultant restreinte aux trois processus concernés en union de S-langages élémentaires : 
$\mathrm{R}=\mathrm{p} 4 \mathrm{p} 4\{\mathrm{p} 5, \mathrm{p} 6\}[\mathrm{p} 5 \otimes \mathrm{p} 6] \mathrm{Up} 4\{\mathrm{p} 4, \mathrm{p} 5, \mathrm{p} 6\}[\mathrm{p} 5 \otimes \mathrm{p} 6] \mathrm{Up} 4\{\mathrm{p} 5, \mathrm{p} 6\}[\mathrm{p} 5 \otimes \mathrm{p} 6] \mathrm{p} 4 \mathrm{U}$ $\mathrm{p} 4 \mathrm{p} 4 \mathrm{p} 5\}[\mathrm{p} 5 \otimes \mathrm{p} 6 \mathrm{p} 6] \mathrm{Up} 4\{\mathrm{p} 4, \mathrm{p} 5\}[\mathrm{p} 5 \otimes \mathrm{p} 6 \mathrm{p} 6] \mathrm{Up} 4 \mathrm{p} 5 \mathrm{p} 5 \mathrm{p} 4 \mathrm{p} 6 \mathrm{p} 6 \mathrm{Up} 4 \mathrm{p} 5 \mathrm{p} 5\{\mathrm{p} 4, \mathrm{p} 6\}$ p6 Up4p5[p5 $\otimes \mathrm{p} 6 \mathrm{p} 6] \mathrm{p} 4$

Choix de représentations : Le langage correspond à 26 situations temporelles possibles entre les procès P4, P5, P6 sur les 409 avant prise en compte des informations temporelles linguistiques utilisées et à $13 * 26 * 13$ (4 394) situations globales entre l'ensemble des 6 procès. D'autres informations temporelles contextuelles peuvent être prises en compte pour réduire l'ensemble des possibilités. Nous avons choisi par exemple la solution par défaut de Battistelli (2000) entre les états, consistant à faire coïncider les trois états. Ce qui réduit l'espace des solutions à 26 . La figure suivante représente deux de ces configurations possibles.

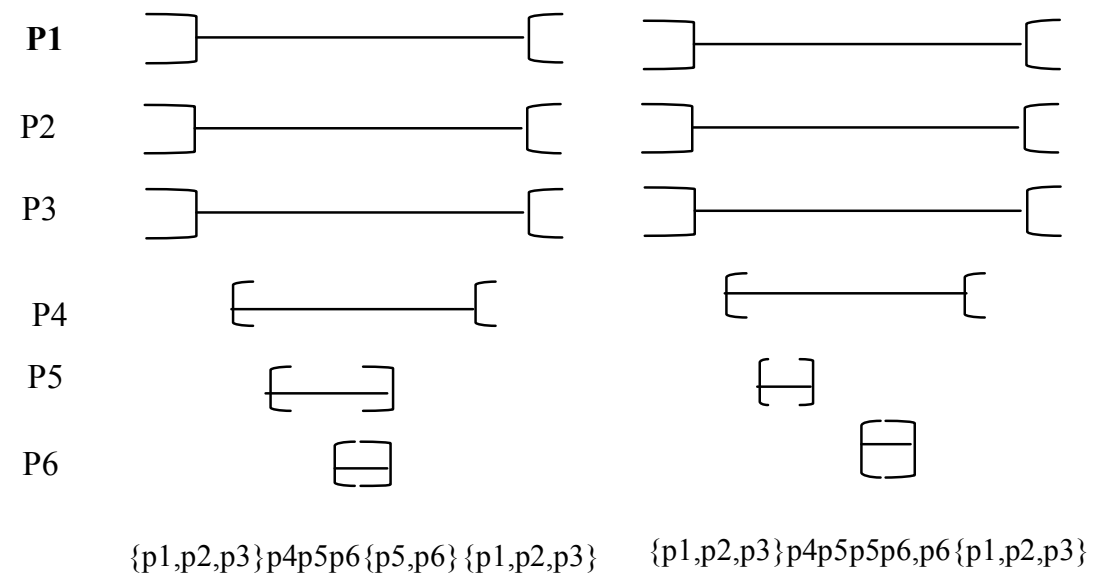

Figure 4 : Deux configurations possibles et leurs S-mots

\section{Conclusion}

Dans cet article nous avons mis en évidence l'intérêt des S-langages pour représenter les informations temporelles linguistiques, souvent largement sous-spécifiées, en illustrant notre démarche dans le modèle de Desclés. Nous nous proposons de suivre la même démarche dans d'autres modèles, et d'étudier systématiquement les parties convexes des treillis correspondant aux S-langages domaines de ces modèles afin d'améliorer la complexité des calculs pour obtenir une implantation utile. 


\section{Références}

Allen J. (1983), Maintaining Knowledge about Temporal Intervals, Communications of the $A C M\}, 26,11,832--843$ Bessière C. \& al (1997), Dossier Raisonnement temporel et spatial, Bulletin de l'AFIA, $\mathrm{n}^{\circ}$ 29.

Autebert, J.-M. (1987\}, Langages Algébriques, Paris, Masson.

Autebert J.-M., Schwer R. S (2003), On generalized Delannoy Paths, SIAM Journal on Discrete Mathematics 16 (2) 208-223.

Balaudé J.-F. et Wolff F eds (2005). Aristote et la pensée du temps, Nanterre, PUPX.

Battistelli D. (2000), Passer du texte à une séquence d'images: analyse spatio-temporelle de textes, modélisation et réalisation informatique (système SPAT), Thèse de doctorat de l'Université Paris IV - Sorbonne, janvier 2000.

Battistelli D., Minel J.-L., Picard E., Schwer S. (2004), «Temporalité linguistique et S-Langages », Actes TALN'04, 19-21 avril 2004, Fès, Maroc.Bras M. (1990), Calcul des structures temporelles du discours thèse de doctorat, Université Paul Sabatier, Toulouse.

Borillo \& al (2003), Tense and Aspect, Handbook of French Semantics, (Corblin F et. de Swart H. eds) CSLI.

Couturat L. (1901), La Logique de Leibniz. Paris, Felix Alcan.

Demirdache H., Uribe-Etxebarria M. (2002) «grammaire des prédicats spatiotemporels : temps, aspects et adverbes de temps », Temps et Aspect, de la morphologie à l'interprétation, Presses Universitaires de Vincennes, 2002.

Desclés J.-P. (1989), « State, Event, Process and Topology " General Linguistics, 29(3), Pennsylvania, State University Press, University Park and London, 159-200.

Desclés J.-P., Guentchéva Z. (2006), Temps et aspects, (à paraître).

Moeschler J. \& al (1998), Le temps des événements. Paris, KIME.

Gosselin L. (1996), Sémantique de la temporalité en français : un modèle calculatoire et cognitif du temps et de l'aspect, Coll. Champs Linguistiques, Duculot.

Kamp H. (1979) « Events, Instants and Temporal Reference », in Semantics from Different points of view, Berlin, Springer Verlag 376-417. 
Kamp H, Reyle U. (1993), From discourse to logic. Kluwer Academic Publisher.

Reichenbach H. (1947), Elements of Symbolic Logic, New York, Free Press.

Russell B. (1929), La méthode scientifique en philosophie (notre connaissance du monde extérieur; Edition française, Vrin.

Saussure L. de (2003), Temps et pertinence, Bruxelles, De Boeck et Larcier.

Schwalb E. et Vila L. (1998), Temporal Constraints : A survey, Constraints 3-2 129-149.

Schwer R. S. (2002), S-arrangements avec répétitions, Comptes Rendus de l'Académie des Sciences de Paris, Série I 334 261-266.

Vet C. (2004) Chronos 2004, conférence invitée, aussi IN (Borillo \& al. 2003) .

Whitehead A. (1920), The concept of nature, Cambridge University Press. 